\title{
Gene Regulation at a Distance in the Epidermal Keratinocyte: The Paradigm of the PADI Gene Locus
}

\author{
Stéphane Chavanas ${ }^{*}, 1,2$, Véronique Adoue ${ }^{1,3}$, Guy Serre ${ }^{1}$ and Michel Simon ${ }^{*}, 1$ \\ ${ }^{1}$ CNRS-University of Toulouse, UMR5165, Toulouse, France \\ ${ }^{2}$ INSERM U563, Purpan University Hospital, Toulouse, France \\ ${ }^{3}$ Departments of Human Genetics and Medical Genetics, McGill University, Montreal, Canada
}

\begin{abstract}
Long-range cis elements are critical regulators of gene transcription, particularly for paralogous genes clustered on a unique chromosomal region. Such are the five $P A D I$ genes in 1p35-36 encoding peptidylarginine deiminases. These enzymes catalyze deimination, or citrullination, a calcium-dependent post-translational modification of proteins which has been implicated in gene regulation and in the pathophysiology of multiple sclerosis and rheumatoid arthritis. The epidermis is an exciting model for understanding PADI gene regulation since only the genes PADI1-3 are expressed in the epidermal keratinocytes, with increased expression levels in the most differentiated cells. Studies on PADI proximal promoters failed to explain such a specificity of expression. Here, we describe long-range regulatory elements identified as essential for the $P A D I 3$ specific expression in differentiated keratinocytes through the formation of a chromatin loop.
\end{abstract}

Keywords: Gene expression, long-range transcriptional regulation, post-translational modification, epidermis.

\section{INTRODUCTION}

The characterization of the genomic elements which govern the spatial and temporal tuning of gene transcription is a key issue in genomic biology, providing for a better understanding of the cell regulatory networks and the functional landscape of the genome [1]. Transcriptional regulation involves the interplay of multiple genomic components, among which cis regulatory segments bind chromatin-modifying enzymes and/or factors interacting with the primary transcription complex. The proximal promoter region of a transcriptional unit barely contains all necessary transcription factor binding modules to direct proper spatiotemporal expression of the cognate gene. The basal transcription machinery, involving the TATA-binding protein and a variety of associated proteins and several additional general transcription factors or coactivators, is hardly sufficient to initiate proper levels of transcription. Additional activators are needed. Moreover, the cognate segments in cis corresponding to the latter may lie at a distance, the transcription control at a distance being now recognized as a critical mechanism of gene regulation [2]. Evolutionary conserved specific binding sites for transcription factors can be found within several hundred or even thousands bases from the transcription initiation site, either upstream or downstream, of the gene to which they are linked. Long-range enhancers have been identified in a variety of chromosomal regions, at a distance up to $1-\mathrm{Mb}$ from their cognate promoters. Distant transcription control rules the expression of essential genes, in particular tandemly organized or clustered genes such as those

*Address correspondence to these authors at the CNRS-University of Toulouse, UMR5165, CHU Purpan, Place du Dr. Balvac, TSA40031, 31059, Toulouse, Cedex 9, France; Te@33 56115 8427; Fax: 335 61499036; E-mails: stephane.chavanas@inserm.fr, michel.simon@udear.cnrs.fr encoding the $\alpha$ and $\beta$ globins, the Th2-type interleukins, and the Hox factors [3-5]. The impairment of long range gene regulation underlies a variety of severe human disorders [2]. Also, transcription control at a distance is thought to play a role in maintaining the physical proximity of genes sharing distant $c i$ s-regulatory elements [6].

Such long-range interactions raise important issue regarding nucleus dynamics. Modifications of the chromatin status and conformation of the chromosome represent two additional levels of regulation. Whatever the intervening genetic distance between distal regulatory elements and cognate promoters, the former should be located in open chromatin regions to allow physical interactions with the corresponding factors. Histone modifications underlie the chromatin conformational changes which make these binding sites accessible or not for their specific regulatory proteins [7]. Also, close proximity between long-range regulatory elements and promoters require chromatin strand bending and/or looping, as evidenced in a number of studies [2].

\section{STRATEGIES TO DISCLOSE DISTANT ENHANCERS}

A difficult step remains the identification of long-range regulatory elements, because they are often developmental stage- and/or tissue-specific. Comparative analysis of human and mouse genomic sequences to highlight highly conserved non-coding sequences (CNS) is a powerful strategy to predict distal candidate regulatory regions [8]. As well, search for DNase I hypersensitive sites indicative of open chromatin regions represents a relevant approach to identify functional regulatory elements. The existence of long-range physical interactions and the juxtaposition of enhancers with promoters can be characterized by the chromosome conformation capture (3C) technique [9]. In 3C, 
formaldehyde is used to cross-link nearby DNA segments, which are eventually digested with a specific restriction enzyme and undergo intramolecular ligation. After reversal of the cross-links, the resulting DNA molecule is a chimera of the two interacting segments. Quantitative PCR assess the frequency of interaction of the two segments. Such intrachromosomal looping has been studied for some genes like these of the beta-globin locus [3].

\section{DISTANT GENE REGULATION IN THE EPIDERMAL KERATINOCYTE}

In the basal keratinocyte, the transcription of the p63 gene is regulated by a keratinocyte-specific enhancer located $160-\mathrm{kb}$ away from it on chromosome $3 \mathrm{q} 28$ [10]. So far this is the most distant gene enhancer identified in the epidermis.

However, most of the key differentiating keratinocyte genes are arranged in conserved segments and appear to undergo coordinated regulation in the course of keratinocyte differentiation: so are the 37 genes within the $5-\mathrm{Mb}$ epidermal differentiation complex (EDC) on 1q21 [11], as well as the genes encoding the desmosomal cadherins, the kallikrein family of serine proteases, and the peptidylarginine deiminases (PADs) family on human chromosomes $18 \mathrm{q} 12,19 \mathrm{q} 13$ and 1p35-36, respectively, albeit the underlying mechanisms are yet poorly documented. Detailed investigation of the $S P R R$ and $P A D I$ loci has provided with important clue to understand long-range regulation. Within the EDC, the 352-kb locus of the genes for the Small PRoline-Rich protein family (SPRR) comprises a set of distal DNase I hypersensitive segments, one of which harbours a strong enhancer activity on the SPRR1A gene promoter located 16-kb away from it [12]. Also, we have recently unravelled a set of distant enhancers within the $P A D I$ gene locus. The PADI locus represents an interesting model to study the spatial and temporal expression of genes in the epidermis given that the PADI gene expression clearly depends upon keratinocyte differentiation state.

\section{REGULATION OF THE PADI GENE LOCUS}

\section{Introduction}

The five PADs constitute a family of enzymes designated PAD1-4 and 6. They are encoded by five paralogous genes (PADI1-4 and 6) clustered within a $355-\mathrm{kb}$ region on chromosome 1p35-36 (Genbank AJ549502) (Fig. 1) [13]. These enzymes are responsible for a $\mathrm{Ca}^{2+}$-dependent posttranslational modification (called deimination or citrullination) whereby the positively charged argininyl residue of a protein is modified into the uncharged citrullinyl residue. Protein deimination has been detected in most mammalian tissues and cells, and PADs are thought to be key actors in severe human diseases. Deimination of myelin basic protein is increased in multiple sclerosis (OMIM 126200) [14].
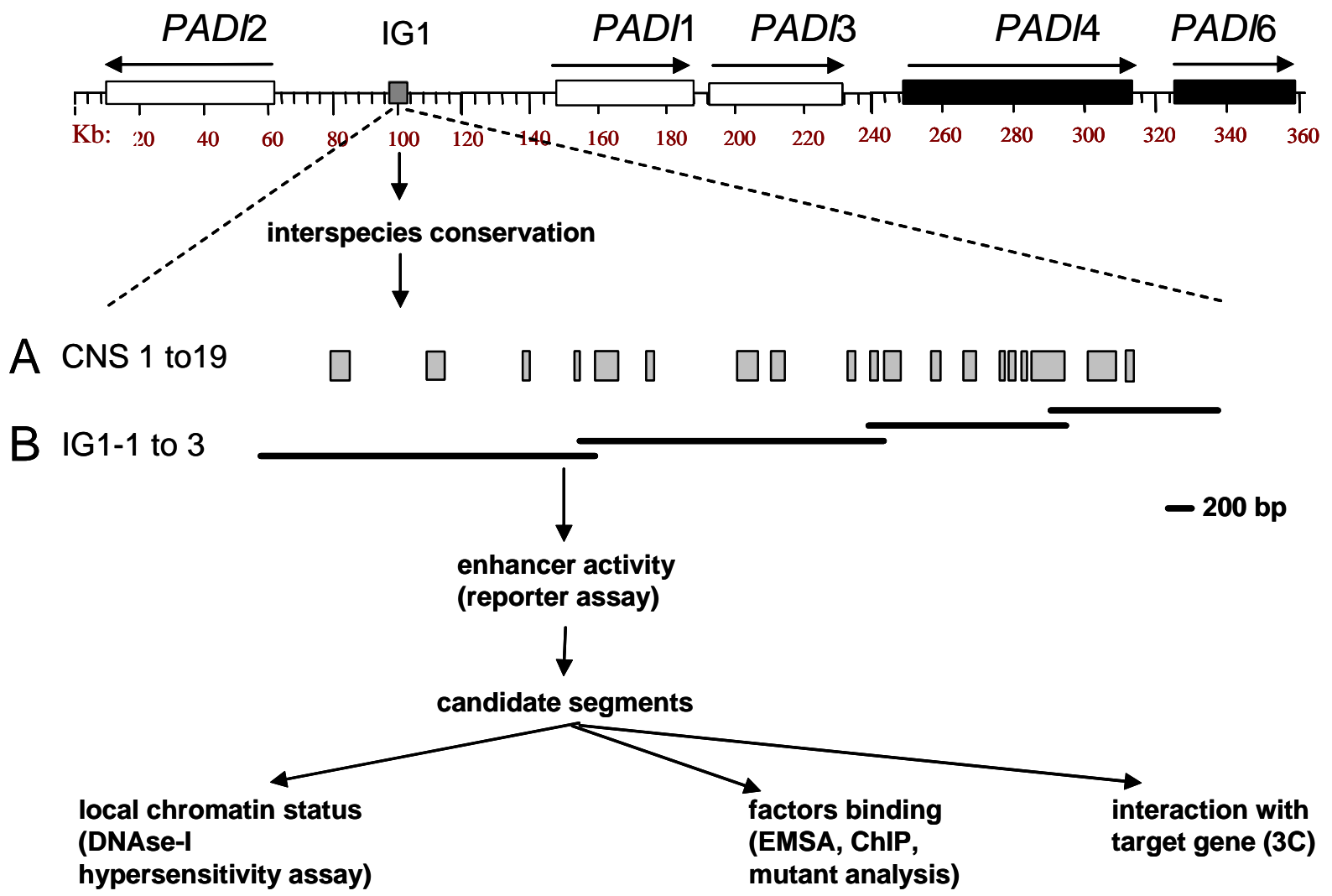

Fig. (1). PADI gene landscape and functional approaches to unravel distant enhancers. Are shown the genes $P A D I 1-3$ expressed in the epidermis (white boxes), the genes PADI4 and 6 with no detectable epidermal expression (black boxes), and the intergenic region IG1 (grey box). (A) Search for evolutionary conservation identified 19 conserved non coding sequences (CNS, grey boxes) along IG1. (B) Restriction analysis identified 4 fragments spanning the whole IG1 region (horizontal bars). Search for enhancer activity in vitro identified candidates which were eventually studied with respect to local chromatin status, nuclear factors binding or level of interaction with target gene. 
Mutations within the gene PADI4 have been shown to be associated with susceptibility to rheumatoid arthritis (OMIM 180300) in Asian populations [15]. Deimination is also a key parameter of epidermis differentiation. A decrease in the levels of deiminated keratins K1 and K10 has been observed in cutaneous diseases, namely bullous congenital ichthyosiform erythroderma (OMIM 113800) [16] and psoriasis (OMIM 177900) [17].

Only products of the $P A D I 1,2$ and 3 genes have been detected in the epidermis [18]. PAD1 is detected throughout the epidermis with an increased expression in differentiated cells, PAD2 is detected in the suprabasal layers, and PAD3 is detected only in the terminally differentiated keratinocytes. The tightly controlled pattern of expression of the PADI genes in the epidermis is consistent with the results obtained in keratinocytes undergoing calcium-stimulated differentiation in vitro. Real-time RT-PCR assays have shown increased expression levels of PADII and PADI3 in cultures of primary Normal Human Epidermal Keratinocytes (NHEKs) differentiated by calcium (Fig. 1). The PADI3 minimal promoter is activated by calcium in the same cells, indicative of transcriptional regulation [19]. PADI1, 2 and 3 gene minimal promoter regions have been characterized by classical approaches [19-21]. Many transcription factors implicated in this proximal regulation are shared by the five $P A D I$ gene promoters: $\mathrm{Sp} 1$ (all four promoters), $\mathrm{Sp} 3$ (PADI2-4) and NF-Y (PADI3-4). Since these transcription factors are not specific to differentiated epidermal layers, proximal regulation alone cannot fully explain the diversity and specificity of $P A D I$ expression in the epidermis.

\section{PADI Locus Genomic Landscape Reveals Candidate Regulatory Regions}

In the search for other PADI transcriptional regulators, we have been interested in non-coding evolutionaryconserved regions. We have assessed overall conservation of the murine and human loci using the program Pip Maker. The dot-plot output has revealed extensive conservation within an 8-kb non-coding region (designated as IG1) located between $P A D I 1$ and $P A D I 2$. IG1 is located at $42 \mathrm{~kb}$ of $P A D I 2$ and $37 \mathrm{~kb}$ of $P A D I 1$, and does not present any known characteristics of a gene (promoter, open reading frame, etc.) (Fig. 1). It clusters nineteen CNS, which present identity rate between mouse and human genomes above $75 \%$, and many potential binding sites for transcription factors. These characteristics support a potential long-range regulatory role of these CNS upon adjacent $P A D I$ gene expression [18].

\section{Three CNS Contain Distant Transcriptional Regulators for the PADI Genes}

To confirm this hypothesis, we first have screened the candidate CNS for activity upon the PADI3 minimal promoter segment in luciferase-based reporter assays in proliferative or differentiating NHEKs [22]. Each of the candidate's CNS has been subcloned in a reporter vector upstream of each of the minimal promoter segments of $P A D I 1,2$ and 3 and that of the SV40 virus.

In the calcium-stimulated cells, we have observed a significantly increased activity of the $P A D I 3$ promoter region and a moderate enhancer effect on the $P A D I 2$ and the SV40 promoter regions in the presence of the CNS2 (63 bp long). The activity of the CNS2 is orientation-independent and copy-number-dependent, and has not been detected in HaCaT, HeLa, A431 or $3 \mathrm{~T} 3$ cells. Thus the CNS2 presents all hallmarks of a transcriptional enhancer and has been named Peptidylarginine deiminase Intergenic Enhancer (PIE). PIE overlaps a DNase I hypersensitive site indicative of chromatin in an active conformation in differentiating NHEKs. This is not the case when chromatin is prepared from proliferative NHEKs.

To investigate the physical proximity between PIE and the $P A D I 3$ gene 5 ' region, the $3 \mathrm{C}$ technique has been used to search for non-random increase in the frequency of contacts between the PADI3 gene promoter and PIE in NHEKs. This has allowed detection of chimeric DNA containing PIE and the $P A D I 3$ promoter region. More, the abundance of such PIE-PADI3 chimeric DNA has been found to be more than 20 -fold greater in differentiating keratinocytes than that in proliferative keratinocytes or dermal fibroblasts, a magnitude greater than any reported so far. In contrast, the ratio between the abundance of PIE-PADI 1 chimeric DNA in unstimulated versus stimulated NHEKs is 9-fold lower than that corresponding to PIE and PADI3 promoter, and is not sensitive to calcium-stimulation. No chimeric DNA could be amplified from the BAC-based control for random association events, or from crude genomic DNA. This indicated non random physical association between specifically PIE and the $P A D I 3$ gene promoter in the nuclei of differentiating NHEKs, through a chromatin looping spanning $86-\mathrm{kb}$.

In conclusion, one enhancer out of 19 candidates within the PADI gene locus has been identified using a candidate approach. The role of the 18 other CNS, should they have any, is unknown. It is possible that they exert a function respective to other $P A D I$ genes, cell types, or stimuli than the ones we have queried. However, the candidate strategy is not appropriate to identify possible enhancers working in combination. This is exemplified by the discovery of two other enhancers within the PADI gene locus, namely PIE $\mathrm{S} 1 / 2$, using a positional approach.

The positional approach, consisting in the study of four restriction fragments encompassing the full length of IG1 was also designed to search for segments/regions with enhancer activity upon the $P A D I 3$ promoter [23]. Four IG1 restriction fragments called IG1-1 to IG1-4 were subcloned upstream the reporter cassette and screened in differentiating or proliferative NHEKs. The constructs containing either the full-length IG1 or any of the IG1-1, -2 or -4 fragments have not shown any significant effect on the $P A D I 3$ promoter. In contrast, the fragment IG1-3 (1764 bp) has displayed a robust and specific enhancer effect (threefold, $\mathrm{P}<0.01$ ) upon $P A D I 3$ and $P A D I 1$ promoters under differentiating conditions. IG1-3 activity is orientation-independent and copy-number-dependent. Eventually we have tested IG1-3 effect on $P A D I 1$ and 3 promoter activity in other cell types, showing that IG1-3 activity was undetectable in other cells than primary normal keratinocytes. To refine the critical enhancer segment within IG1-3, a series of deletion mutants have been engineered and tested for their ability to enhance $P A D I 3$ promoter activity in luciferase assays. We have 
identified two minimal regions of 346 and $245 \mathrm{bp}$ located in IG1-3, 1-kb distant from each other, which appear both to be necessary to the IG1-3 enhancer activity in differentiated NHEKs. We have named these two sequences PAD Intergenic Enhancer supplementary Segment 1 (PIE-S1) and 2 (PIE-S2), respectively. PIE-S1 and PIE-S2 overlap the CNS10 and CNS14. Neither CNS10 nor CNS14 show any detectable effect when assayed individually. PIE-S1 and PIE-S2 have been found to be hypersensitive, with a dramatic fall in the remaining copy number in calciumstimulated NHEKs. This indicates an open chromatin conformation and supports their role as enhancers prone to bind transcriptional activators in differentiated keratinocytes. Unexpectedly, we have also found DNase I hypersensitivity of PIE-S1 and PIE-S2 in unstimulated keratinocytes, that is in conditions where no detectable and significant enhancer effect has been observed.

In conclusion, the positional approach identified a bipartite enhancer of $P A D I 3$ promoter activity constituted by PIE-S1 and PIE-S2, each being 83 and $39 \mathrm{~kb}$ away from $P A D I 3$, respectively.

\section{Transcription Factors Interplay Mediates PADI3 Enhancer Activity}

As has been revealed by electrophoretic mobility shift assays, chromatin immunoprecipitation (ChIP) assays and functional assays using dominant negative mutants or chemical inhibitors, binding of the two AP-1 family factors c-Jun and c-Fos to PIE is required for its activity. The AP-1 family of transcription factors mainly consists of dimers of c-fos, Fos-B, Fra-1, Fra-2, Jun-B, Jun-D, and c-Jun. Each factor is differentially expressed depending on the keratinocyte differentiation state. AP-1 transcription factors play a major role in skin physiology [24]. In particular they regulate genes specific to differentiated keratinocytes, e.g. those for filaggrin, involucrin, SPRR, transglutaminase 1, caspase 14, and loricrin [25-28]. A significantly increased frequency of binding was found in differentiating versus proliferative NHEKs (2.9 and 2.8 -fold, respectively). Blocking c-Jun by co-transfection with a dominant negative mutant (TAM67) impairs the enhancer effect of PIE. Treatment of stimulated NHEKs with nordihydroguaiaretic acid, a drug known to reduce c-Fos protein levels and to inhibit binding of the Fos-Jun dimer to DNA, results in a dramatic decrease of $P A D I 3$ mRNA levels, as measured by real-time RT-PCR. Altogether these results show that c-Jun and c-Fos are recruited at PIE and that c-Jun at least is essential for its activity. This is consistent with the reported increased expression of c-Jun and c-Fos in the epidermal granular layer. Since the basal activity of the $P A D I 3$ minimal promoter requires binding of the transcription factors NF- Y and $\mathrm{Sp} 1 / 3$ [22], and in order to know if they are necessary for PIE activity, we have mutated the two CCAAT boxes or the two GC-boxes, their respective binding sites, and proceeded to luciferase assays. Invalidation of the CCAAT boxes dramatically decreases the PIE enhancer effect on PADI3 promoter. However, mutation of the GC-boxes does not change PIE effect. These results suggest a possible functional interaction between PIE and the CCAAT boxes, possibly through c-Jun/NF-Y association, as reported for other genes.
Because of our findings respective to the DNAse I hypersensitive status of PIE-S1/2, we have queried which transcription factors bind to PIE-S1 and -S2 in both proliferative and differentiated conditions. Sequence analysis has revealed several conserved potential binding sites for the transcription factors Sp1, NF1 and MIBP1/RFX1 in PIE-S1, and AP-1 in PIE-S2. Functional assays have shown that both AP-1 and MIBP1/RFX1 binding sites are essential for the IG1-3 enhancer activity. RFX1 belongs to a conserved family of five homo- and hetero-dimeric proteins with DNAbinding properties. RFX1 and MIBP1 are known to interact in vivo and to be involved in the expression of a variety of cellular and viral genes, including c-myc, major histocompatibility complex class II and human hepatitis B virus genes. An association between MIBP1/RFX1 and AP-1 in the regulation of a gene, namely MHC class II genes, has already been reported. MIBP1 mRNA has been detected in epidermal granular keratinocytes, and we have successfully detected both MIBP1 and RFX1 transcripts in calciumdifferentiated NHEKs [23]. Quantitative RT-PCR assays have not revealed any differences on their expression levels between proliferative and differentiated NHEKs. However, we have failed to detect any fixation of MIBP1/RFX1 onto PIE-S1 by EMSA, possibly because of poor reactivity of the antibody in the experimental conditions. Besides we have revealed an increased occupancy of PIE-S2 by c-Jun (4.6 \pm 0.8 fold) in differentiated compared to proliferative keratinocytes. We have also found an increased occupancy of PIE-S2 by JunD (7.3 \pm 2 fold) in proliferative compared to differentiated keratinocytes. Taken together, these data suggest that PIE-S2 bound distinct AP-1 factors according to the keratinocyte differentiation state: JunD in the proliferative condition showing no enhancer activity and cJun in the differentiating condition showing significant enhancer activity. This was consistent with the fact that JunD is mainly expressed in the basal layer of the epidermis containing proliferative keratinocytes, and c-Jun in the most differentiated keratinocytes [24]. The ratio of c-Jun/JunD has previously been proposed as a key parameter of gene expression and growth regulation in other cell types, i.e. small intestinal crypt cells, NIH-3T3 fibroblasts and malignant fibrosarcoma cells [29-31]. This has generally been explained through the regulation of genes with opposite effects. Our results were the first description of a competition between c-Jun and JunD on the same AP-1 site as an explanation to the mode of action of the c-Jun/JunD ratio.

\section{CONCLUSION}

Increasing evidence for the importance of distant interactions between DNA segments provides with an appreciation of the complexity of gene regulation. Long range associations allow enhancers to interact in close vicinity to the target promoter to stimulate transcription. Our findings disclose that transcriptional regulation of the PADI genes involves additional modules other than those within the proximal promoters, what can explain the specificity of the PADI gene expression in differentiated keratinocytes. We have highlighted long-distance action of three evolutionary conserved DNase I hypersensitive sites, namely PIE, PIE-S1 and PIE-S2 on the regulation of PADI 1 and PADI3, involving c-Jun, c-Fos, JunD and MIBP1/RFX1 factors (Fig. 
A

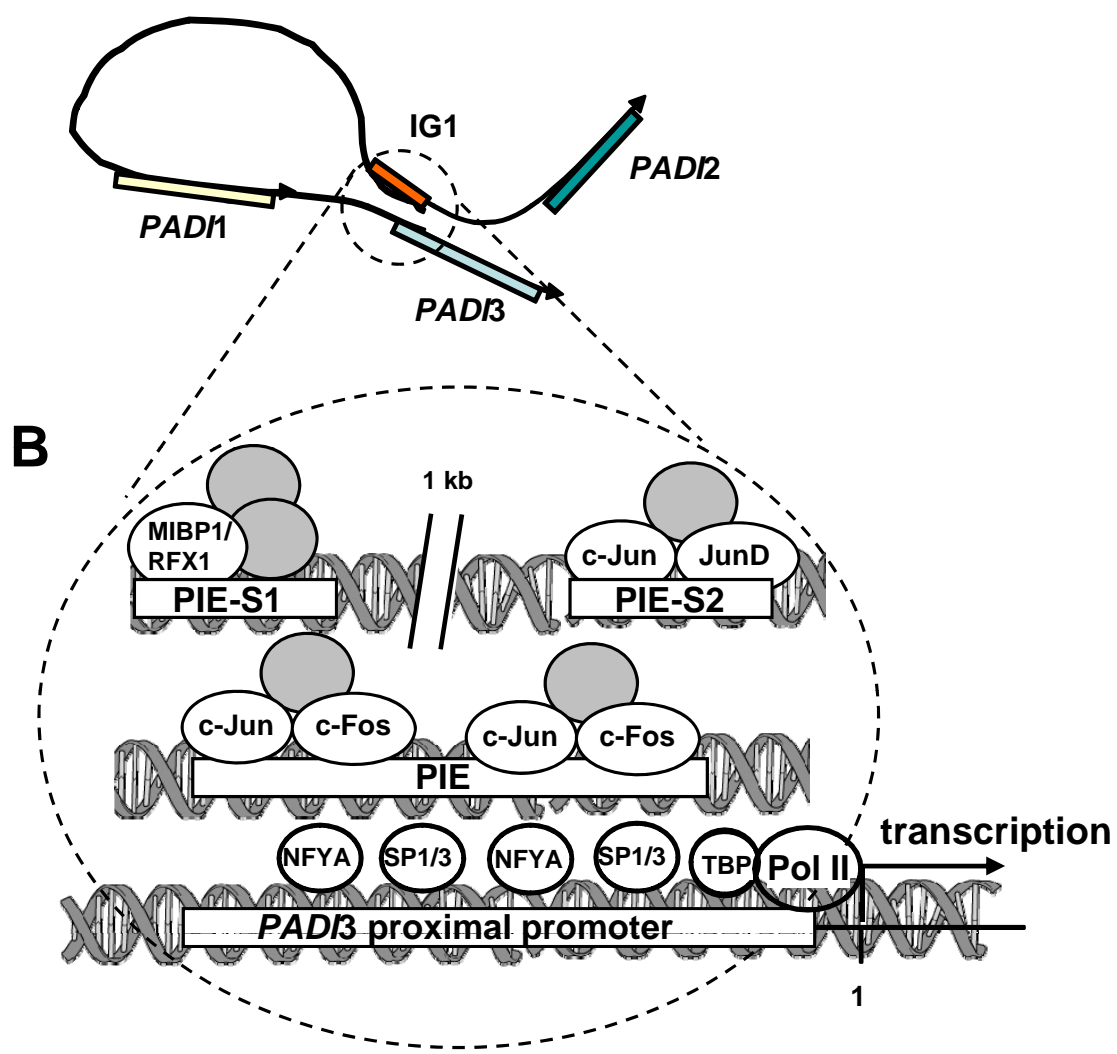

Fig. (2). Hypothetical model of the PADI3 transcriptional regulation in keratinocyte. (A) Shown is the hypothetical chromatin loop underlying $P A D I 3$ transcription control at the chromosomal scale, as assessed by $3 \mathrm{C}$. Note IG1 interacting with $P A D I 3$ gene promoter. IG1 is $\sim 8 \mathrm{~kb}$-long and is located $86 \mathrm{~kb}$ away from the $P A D I 3$ gene. (B) Shown are the hypothetical events underlying $P A D I 3$ transcription control at the molecular level. Part of the PADI gene locus including the intergenic region IG1 is shown. IG1 contains three long-distance enhancers, namely PIE, PIE-S1 and PIE-S2. In calcium-stimulated keratinocytes, the binding of c-Jun and/or c-Fos dimers to both AP-1 sites of PIE is enhanced. This in turn increases the transcription of PADI3 (broken arrow), possibly through molecular interactions involving known (open circles) or yet unknown (grey circles) transcription factors. The high calcium concentration also favours the binding of c-Jun but not JunD to PIE-S2. Cooperation between PIE-S2 and PIE-S1, two sites located $1 \mathrm{~kb}$ apart, also induces PADI3 gene expression. Transcription factors (cJun, c-Fos, JunD, NF-Y and Sp1/3), the TATA-box binding protein (TBP) and RNA polymerase II (PolII) are shown as open circles, others possible interacting factors are in grey.

2). This is an essential step toward better knowledge of PAD expression in the epidermis. A transgenic mouse model could definitely elucidate the role of PIE and PIE-S1/-S2 in vivo. Highlighting IG1 as a genomic region involved in the transcriptional control of $P A D I$ genes may also be critical to understanding PAD implication in the pathophysiology of diseases characterized by modifications in the deiminated protein rate. Indeed, recent works have evidenced an association between acute myeloid leukemia and a singlenucleotide polymorphism within a distal enhancer of the PU.1 gene [32] and have identified single-nucleotide polymorphisms in tissue-specific long-range enhancers as risk factors for breast cancer [33]. PIE, PIE-S1, PIE-S2 and other conserved non coding segments of IG1 can be reasonably considered as candidates in association studies for genetically determined diseases related to deimination, including rheumatoid arthritis and multiple sclerosis. Last, loss-of-function polymorphisms in the filaggrin gene associate with atopic disease [34]. Given the massive deimination of filaggrin polypeptides during keratinocyte differentiation, we assume that any regulators of PAD gene expression, including IG1, are possible candidates for increased susceptibility to atopy.

\section{ACKNOWLEDGEMENTS}

We are indebted to Catherine Bouchouata for her technical assistance. The authors would like to thank $\mathrm{Pr}$ Hidenari Takahara and co-workers for their contributions to the research discussed here. The author laboratory is supported partly by the CNRS, the University of Toulouse III, the INSERM, the European Social Funds, the European Regional Development Funds, the French Society of Dermatology (SFD), the Society for Dermatological Research (SRD) and Pierre Fabre Dermo-Cosmétique.

\section{REFERENCES}

[1] The ENCODE project. Identification and analysis of functional elements in $1 \%$ of the human genome by the ENCODE pilot project. Nature 2007; 447: 799-816. 
[2] Kleinjan DA, van Heyningen V. Long-range control of gene expression: emerging mechanisms and disruption in disease. Am J Hum Genet 2005; 76: 8-32.

[3] Li Q, Peterson KR, Fang, X, Stamatoyannopoulos G. Locus control regions. Blood 2002; 100: 3077-86.

[4] Loots GG, Locksley RM, Blankespoor CM, et al. Identification of a coordinate regulator of interleukins 4,13 , and 5 by cross-species sequence comparisons. Science 2000; 288: 136-40.

[5] Santini S, Boore JL, Meyer A. Evolutionary conservation of regulatory elements in vertebrate Hox gene clusters. Genome Res 2003; 13: 1111-22.

[6] Waterston RH, Lindblad-Toh K, Birney E, et al. Initial sequencing and comparative analysis of the mouse genome. Nature 2002; 420: 520-62.

[7] Jenuwein T, Allis CD. Translating the histone code. Science 2001; 293: 1074-80.

[8] Pennacchio LA, Rubin EM. Genomic strategies to identify mammalian regulatory sequences. Nat Rev Genet 2001; 2: 100-9.

[9] Dekker J, Rippe K, Dekker M, Kleckner N. Capturing chromosome conformation. Science 2002; 295: 1306-11.

[10] Antonini D, Rossi B, Han R, et al. An autoregulatory loop directs the tissue-specific expression of p63 through a long-range evolutionarily conserved enhancer. Mol Cell Biol 2006; 56: 330818.

[11] Marenholz I, Zirra M, Fischer DF, Backendorf C, Ziegler A, Mischke D. Identification of human epidermal differentiation complex (EDC)-encoded genes by subtractive hybridization of entire YACs to a gridded keratinocyte cDNA library. Genome Res 2001; 11:341-55.

[12] Martin N, Patel S, Segre JA. Long-range comparison of human and mouse Sprr loci to identify conserved noncoding sequences involved in coordinate regulation. Genome Res 2004; 14: 2430-8.

[13] Chavanas S, Mechin M, Nachat R, et al. Peptidylarginine deiminases and deimination in biology and pathology: relevance to skin homeostasis. J Dermatol Sci 2006; 44: 63-72.

[14] Moscarello MA, Pritzker L, Mastronardi FG, Wood DD. Peptidylarginine deiminase: a candidate factor in demyelinating disease. J Neurochem 2002; 81: 335-43.

[15] Suzuki A, Yamada R, Chang X, et al. Functional haplotypes of PADI4, encoding citrullinating enzyme peptidylarginine deiminase 4, are associated with rheumatoid arthritis. Nat Genet 2003; 34: 395-402.

[16] Ishida-Yamamoto A, Senshu T, Eady RA, et al. Sequential reorganization of cornified cell keratin filaments involving filaggrin-mediated compaction and keratin 1 deimination. J Invest Dermatol 2002; 118: 282-7.

[17] Ishida-Yamamoto A, Senshu T, Takahashi H, Akiyama K, Nomura $\mathrm{K}$, Iizuka $\mathrm{H}$. Decreased deiminated keratin $\mathrm{K} 1$ in psoriatic hyperproliferative epidermis. J Invest Dermatol 2000; 114: 701-5.

[18] Chavanas S, Mechin MC, Takahara H, et al. Comparative analysis of the mouse and human peptidylarginine deiminase gene clusters reveals highly conserved non-coding segments and a new human gene, PADI6. Gene 2004; 330: 19-27.

[19] Dong S, Kanno T, Yamaki A, et al. NF-Y and Sp1/Sp3 are involved in the transcriptional regulation of the peptidylarginine deiminase type III gene (PADI3) in human keratinocytes. Biochem J 2006; 397: 449-59.

[20] Dong S, Kojima T, Shiraiwa M, et al. Regulation of the expression of peptidylarginine deiminase type II gene (PADI2) in human keratinocytes involves $\mathrm{Sp} 1$ and Sp3 transcription factors. J Invest Dermatol 2005; 124: 1026-33

[21] Dong S, Ying S, Kojima T, et al. Crucial Roles of MZF1 and Sp1 in the Transcriptional Regulation of the Peptidylarginine Deiminase Type I Gene (PADI1) in Human Keratinocytes. J Invest Dermatol 2007; 128(3): 549-57.

[22] Chavanas S, Adoue V, Mechin MC, et al. Long-range enhancer associated with chromatin looping allows AP-1 regulation of the peptidylarginine deiminase 3 gene in differentiated keratinocyte. PLoS One 2008; 3: e3408.

[23] Adoue V, Chavanas S, Coudane F, et al. Long-range enhancer differentially regulated by c-Jun and JunD controls peptidylarginine deiminase-3 gene in keratinocytes. J Mol Biol 2008; 384: 1048-57.

[24] Angel P, Szabowski A, Schorpp-Kistner M. Function and regulation of AP-1 subunits in skin physiology and pathology. Oncogene 2001; 20: 2413-23

[25] Crish JF, Zaim TM, Eckert RL. The distal regulatory region of the human involucrin promoter is required for expression in epidermis. J Biol Chem 1998; 273: 30460-5.

[26] Jang SI, Steinert PM. Loricrin expression in cultured human keratinocytes is controlled by a complex interplay between transcription factors of the Sp1, CREB, AP1, and AP2 families. J Biol Chem 2002; 277: 42268-79.

[27] Jang S-I, Steinert PM, Markova NG. Activator protein 1 activity is involved in the regulation of the cell type-specific expression from the proximal promoter of the human profilaggrin Gene. J Biol Chem 1996; 271: 24105-14.

[28] Rossi A, Jang SI, Ceci R, Steinert PM, Markova NG. Effect of AP1 transcription factors on the regulation of transcription in normal human epidermal keratinocytes. J Invest Dermatol 1998; 110: 3440 .

[29] Bossy-Wetzel E, Bravo R, Hanahan D. Transcription factors junB and $\mathrm{c}$-jun are selectively up-regulated and functionally implicated in fibrosarcoma development. Genes Dev 1992; 6: 2340-51.

[30] Patel AR, Wang JY. Polyamine depletion is associated with an increase in JunD/AP-1 activity in small intestinal crypt cells. Am J Physiol 1999; 276: G441-50.

[31] Pfarr CM, Mechta F, Spyrou G, Lallemand D, Carillo S, Yaniv M. Mouse JunD negatively regulates fibroblast growth and antagonizes transformation by ras. Cell 1994; 76: 747-60.

[32] Steidl U, Steidl C, Ebralidze A, et al. A distal single nucleotide polymorphism alters long-range regulation of the PU.1 gene in acute myeloid leukemia. J Clin Invest 2007; 117: 2611-20.

[33] Yang R, Frank B, Hemminki K, et al. SNPs in ultraconserved elements and familial breast cancer risk. Carcinogenesis 2008; 29: 351-5.

[34] Sandilands A, Terron-Kwiatkowski A, Hull PR, et al. Comprehensive analysis of the gene encoding filaggrin uncovers prevalent and rare mutations in ichthyosis vulgaris and atopic eczema. Nat Genet 2007; 39: 650-4. 